\title{
Studies on the Enamel Structure of Transplanted Tooth Germ
}

\author{
Hitoshi Yamamoto ${ }^{1}$, Jinglei Chai ${ }^{2}$, Kunihiro Suzuki ${ }^{1}$, Rumi Yokota ${ }^{1}$, Hideki Chisaka ${ }^{1}$, Toshiro Sakae', \\ Han-Sung Jung ${ }^{2}$, Yukishige Kozawa ${ }^{1}$
}

\begin{abstract}
1) Department of Histology, Cytology and Developmental Anatomy, Nihon University School of Dentistry at Matsudo, 870-1 Sakaecho Nishi 2, Matsudo, Chiba 270-8587, Japan

2) Division in Anatomy and Developmental Biology, Department of Oral Biology, College of Dentistry, Yonsei University, 134 Shinchondong, Soedaemun-gu, Seoul 120-749, Korea
\end{abstract}

\begin{abstract}
Enamel covers the dentin in mammalian teeth. It is believed that the enamel structure reflects the function of teeth, since the enamel structure is different depending on an animal's feeding habits. Tooth germ transplantation is a popular method for developmental and tissue engineering research. However, there is little information about the structure of the enamel of transplanted tooth germ, although tooth structure, especially enamel structure, is very important for the function of teeth. In this study, the enamel structure of E13.5 mouse mandible first molars transplanted into the kidney capsule was observed by scanning electron microscopy. Many part of the tooth crown are formed at transplantation 7 day. Although enamel and dentin were observed at the cusp portion, hard tissue was not formed at the cervical portion. The enamel prisms were observed at day 21 after transplantation. The enamel was divided into three layers according to the running pattern of the enamel prisms as normal tooth germ in vivo. However, the width of the various layers was different. It seems likely that the microenvironment surrounding tooth germ may play an important role in determining the structure of the enamel, since normal tooth germ grows in the calcified alveolar bone according as development.
\end{abstract}

Key words: enamel, transplantation, mouse

\section{Introduction}

Enamel covers the crown dentin of mammalian teeth. The basic unit of enamel is enamel prism, and many structures such as Hunter-Schreger bands are observed in the enamel caused by the running pattern of the enamel prisms. It is generally believed that this running pattern of the enamel prisms reflects the function of the tooth, since the enamel structure of animals is different depending on the animal's feeding habits ${ }^{1}$.

Recently, many investigators have focused on tooth development and tooth tissue engineering due to the social need for advances in these fields requirement. Tooth germ culture and tooth germ transplantation are popular methods for such research. For tooth germ culture, modified methods such as the floating method $^{2}$, have been developed to prevent morphological changes in culture tooth germ. Moreover, novel in vitro culture system for tooth root development was designed ${ }^{3}$, although it is difficult to create an in vitro culture to examine root formation, because the tooth root grows in a special environment surrounded by calcified alveolar bone ${ }^{4}$. On the other hand, tooth germs were transplanted into subcutaneous tissue ${ }^{5}$, kidney capsule ${ }^{6,7}$, spleen $^{8}$ and so on. However, there is little information about the tooth structure, especially the enamel structure, of transplanted tooth germs. Therefore, the authors attempted to clarify the enamel structure of the transplanted tooth germ by scanning electron microscope.

\section{Materials and methods}

Fetal mice on prenatal day 13.5 (E13.5) were removed from pregnant mice and slaughtered by decapitation under deep anesthesia with diethyl ether. Mandible first molars were dissected

Correspondence to Hitoshi Yamamoto,Department of Histology, Cytology and Developmental Anatomy, Nihon University School of Dentistry at Matsudo, 870-1 Sakaecho Nishi 2, Matsudo, Chiba 270-8587, Japan. Tel: +81-47-360-9323, Fax: +81-47-360-9324 out and immediately transplanted into the kidney capsule. Host mice were perfused with $4 \%$ paraformaldehyde in $0.1 \mathrm{M}$ phosphate buffer (pH 7.4) after transplantation 7, 14, 21 days. The removed kidney was immersed in the same fixative for an additional 2-3 days at $4^{\circ} \mathrm{C}$. Then, the transplanted tooth germs were removed from the kidney. After optical microscopic observation, specimens were embedded in polyester resin and polished medial-distally until a miller surface was achieved with diamond paste. Then, specimens were etched by $0.5 \%$ hydrochloride solution for 30 seconds. After critical point drying and Au coating, observation was made of the ground surface by means of scanning electron microscopy (SEM).

\section{Results}

At transplantation day 7, transplanted tooth germ closely resembled normal mandible first molar (Fig. 1) Hard tissues, enamel and dentin, were not observed at cervical portion, although cell elements of tooth germ, i.e., inner enamel epithelium, odontoblasts and other pulp cells, were recognized (Fig. 2). In contrast, the enamel and the dentin were formed at the cusp portion of the transplanted tooth germ (Fig. 3). Moreover, the enamelfree area, a specific structure of the rodent molar, was observed at the cusp tip (Fig. 3).

At transplantation day 14, the transplanted tooth germ grew toward both the lingual-buccal and medial-distal directions (Fig. 4 ). The crown formation was finished and the tooth root was observed in the transplanted tooth germ (Fig. 5). The enamel was formed from the cusp portion to the cervical portion. The width of enamel at this stage was thicker than that after transplantation day 7 (Fig. 5). However, enamel prisms were not observed anywhere in the enamel (Fig. 6).

At transplantation day 21, the lingual-buccal width was extended, although the medial-distal length was not obviously changed (Fig. 7). The tooth root formation advanced (Fig. 8). Fig. 
9 and Fig. 10 show different areas of the transplanted tooth enamel. In Fig. 9, the enamel prisms were clearly observed and the enamel was divided into three layers by the running pattern of the enamel prisms. The enamel prisms ran parallel to each other in the surface layer of the enamel, and in a complicated manner and formed a special pattern in the middle portion of the enamel. Moreover, the enamel prisms were shown to be opaque in most of the inner part of the enamel (Fig. 9). However, the width of each layer was different from that of the layers in the normal enamel structure in $v_{i v o}$. In Fig. 10, the running pattern of the enamel prisms in the middle area was irregular. However, this pattern was also similar to that of the normal tooth germ ${ }^{9}$.

\section{Discussion}

A previous study showed that the enamel of the mouse mandible first molar in vivo is divided into three layers by the running pattern of the enamel prisms ${ }^{9}$. This study showed the same results in an in vivo study. However, the width of each layer of the transplanted tooth germ was different from that of normal tooth germ in vivo. It is generally known that tooth germ grows in the calcified alveolar bone as developmental process. Moreover, studies using ordinary tooth germ culture methods have failed to observe root formation due to the presence of calcified alveolar bone in the mandible $e^{4}$. On the other hand, it was reported that the running pattern of the enamel was formed by the positive interaction of ameloblasts and
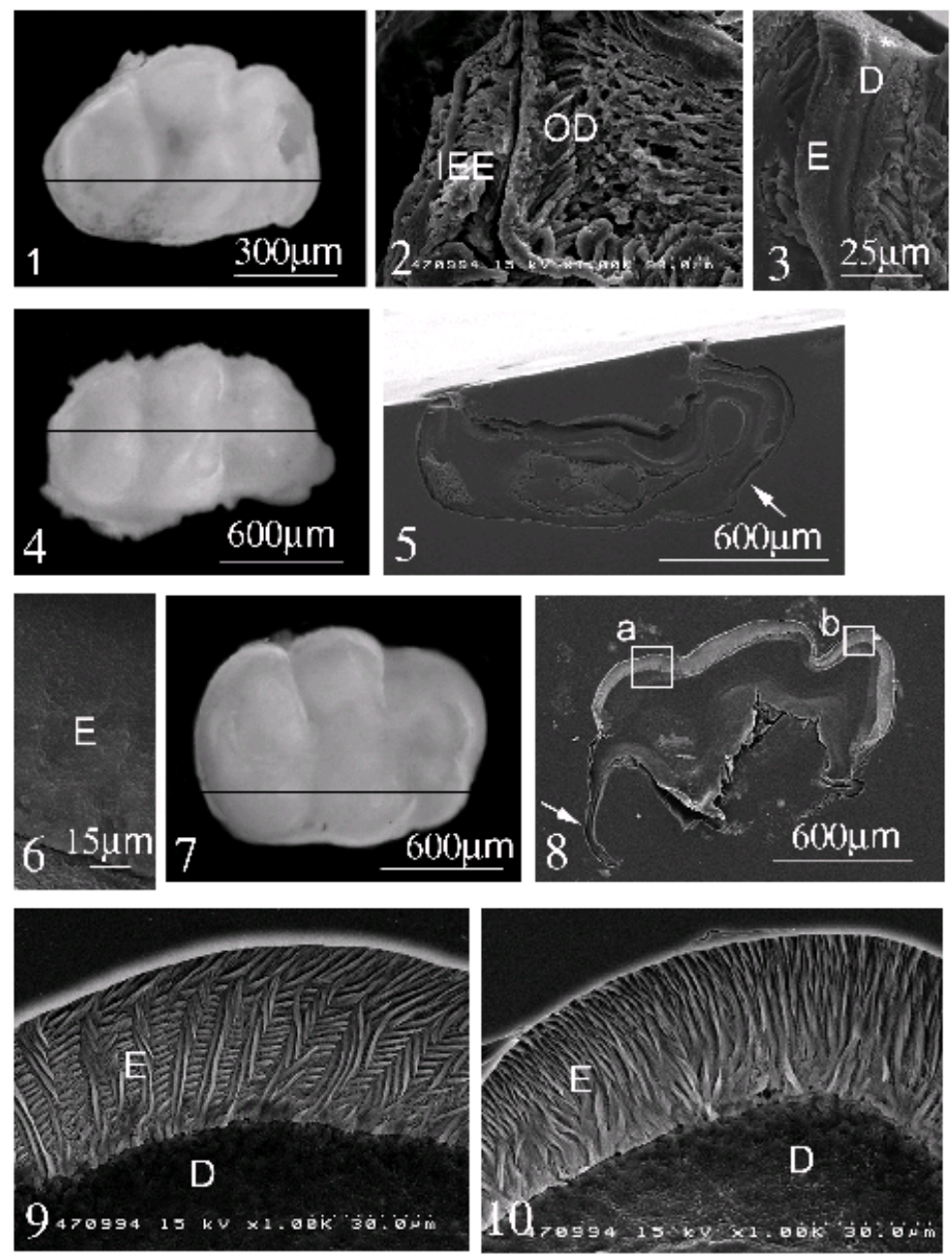

Fig. 1 shows an occlusal view of transplanted tooth germ by optical microscope at transplantation day 7. The black line shows the grinding plane. Inner enamel epithelium (IEE) and odontoblasts (OD) are observed at the cervical portion (Fig. 2), although enamel (E) and dentin (D) are formed at the cusp tip (Fig. 3). Moreover, an enamel-free area (asterisk) exists at the cusp tip. Fig. 4 shows an occlusal view of transplanted tooth germ by optical microscope at transplantation day 14 . The tooth germ grows in both the lingual-buccal and medial-distal directions. The black line shows the grinding plane. Fig. 5 shows a SEM image of the ground surface. Tooth formation already begins to occur at this stage (Fig. 5 arrow). The enamel prisms are not observed in whole enamel (Fig. 6). Fig. 7 shows an occlusal view obtained by optical microscopy at transplantation day 21. the black line shows the grinding plane. The tooth root formation has advanced (Fig. 8 arrow). Fig. 9 and Fig. 10 are higher magnification of "a" and "b” in Fig. 8 respectively. The enamel is divided into three layers by the running pattern of the enamel prisms (Fig. $9,10)$.

D: dentin, E: enamel 
International symposium of Maxillofacial \& Oral Regenerative Biology in Okayama 2005

the whole enamel organ ${ }^{10,11}$. These findings suggest that the inside of calcified alveolar bone has a specific microenvironment and this microenvironment may control the movement of ameloblasts and the whole enamel organ.

In previous transplantation studies, there was no discussion about the tooth shape of transplanted tooth germs. Since tooth germs fell down according as hard tissue formation and the morphology of tooth germ affected for this accident in long-term tooth organ culture, a floating method was designed ${ }^{2}$. However, there is little information about the transplanted tooth germ, since many investigators did not focus on the tooth structure. In the present study, the growth in the lingual-buccal direction occurred later than that in the medial-distal direction. It is supposed that the tension between the kidney capsule and kidney cortex acted as a lingual-buccal axis in this study, and the shape of the tooth germs might have been influenced by the direction of tooth germ. Therefore, when tooth germs are transplanted, attention must be paid the direction of the tooth germ.

The blood supply is very important for transplantation, as it provides nutrition. It is likely that the condition of the transplanted tooth germ is more strongly affected by the environment of the host compared with other types of tissue transplantation, since enamel, dentin and cementum calcify during development. In this study, the kidney capsule was selected as the host tissue, and histological examination were performed. The authors intend to analyze the chemical elements, hardness, fine structure of other hard tissues and differences between host tissues after the transplantation.

\section{Acknowledgements}

This study was supported by grants-in aid for Scientific Research from the Japan Society for the Promotion of Science (No. 16591840, No. 16791115), Nihon University Individual Research Grant for (2004) (No. 04-124), a Grant-in-Aid for “Academic Frontier” Project from MEXT (2003-2007) and a Grant from the Ministry of Education, Culture, Sports, Science and Technology to Promote 2001-Multidisciplinary Research Projects (in 2001-2005).

\section{References}

1) Lester KS., Hand SJ. and Vincent F.: Adult phyllostomid (bat) enamel by scanning electron microscopy-with a note on dermopteran enamel. Scanning Microsc, 2: 371-383, 19988

2) Sakakura Y.: A new culture method assuring the threedimensional development of mouse embryonic molar tooth in vitro. Calcif Tissue Int, 39: 271-278, 1986

3) Yamamoto H., Cho SW., Kim EJ., Kim JY., Fujiwara N. and Jung HS.: Developmental properties of the Hertwig's epithelial root sheath in mice. J Dent Res, 83: 688-692, 2004

4) Bernstein AB., Preisig E. and Schroeder HE.: In-vitro formation of a collagenous matrix upon previously diseased and experimentally treated cemental root surfaces. Cell Tissue Res, 259: 603-606, 1990

5) Isogawa N., Terashima T., Nakano Y., Kindaichi J., Takagi Y. and Takano Y.: The induction of enamel and dentin complexes by subcutaneous implantation of reconstructed human and murine tooth germ elements. Arch Histol Cytol, 67: 65-77, 2004

6) Yamamoto H., Kim EJ., Cho SW. and Jung HS.: Analysis of tooth formation by reaggregated dental mesenchyme from mouse embryo. J Electron Microsc, 52: 559-566, 2003

7) Cho SW., Hwang HJ., Kim JY., Song WC., Song SJ., Yamamoto H. and Jung HS.: Lineage of non-cranial neural crest cell in the dental mesenchyme: using a lacZ reporter gene during early tooth development, J Electron Microsc, 52: 567-571, 2003

8) Ishizeki K., Nawa T. and Sugawara N.: Calcification capacity of dental papilla mesenchymal cells transplanted in the isogenic mouse spleen. Anat Rec, 226: 279-287, 1990

9) Lyngstadaas SP., Moinichen CB. and Risnes S.: Crown morphology, enamel distribution, and enamel structure in mouse molars. Anat Rec, 250: 268-280, 1998

10) Hanaizumi Y., Shimokobe H. and Wakita M.: The threedimensional structure of Tomes' processes and their relationship to arrangement of enamel prism in dog teeth, Arch Histol Cytol, 57: 129-138, 1994

11)Kozawa Y.: Development of mammalian dentition and cytological base of enamel development, Archives of Comparative Biology of Tooth Enamel, 8: 107-127, 2003 (in Japanese) 